\title{
Erratum: Effects of Electron Irradiation During the Growth of ITO Films by an Using RF Sputtering System
}

\author{
[J. Korean Phys. Soc. 69, 1236 (2016)] \\ Young Joon Yoon, ${ }^{*}$ Sung Hwan CHO and Bongho KIM \\ Nano-Convergence Materials Center, Korea Institute of Ceramic Engineering and Technology, Jinju 52851, Korea
}

DOI: $10.3938 / \mathrm{jkps} .72 .196$

The title "Effects of Electron Irradiation During the Growth of ITO Films by an Using RF Sputtering System" had a typing error. It should be written as "Effects of Electron Irradiation during the Growth of ITO Films by Using an RF sputtering System".

*E-mail: yjyoon@kicet.re.kr; Fax: +82-55-792-2651 